\title{
Recent Progress in Presolar Grain Studies
}

\author{
Sachiko Amari \\ McDonnell Center for the Space Sciences and the Physics Department, Washington University, \\ One Brookings Drive, Saint Louis, Missouri 63130-4899, USA
}

\begin{abstract}
Presolar grains are stardust that condensed in stellar outflows or stellar ejecta, and was incorporated in meteorites. They remain mostly intact throughout the journey from stars to the earth, keeping information of their birthplaces. Studies of presolar grains, which started in 1987, have produced a wealth of information about nucleosynthesis in stars, mixing in stellar ejecta, and temporal variations of isotopic and elemental abundances in the Galaxy. Recent instrumental advancements in secondary ion mass spectrometry (SIMS) brought about the identification of presolar silicate grains. Isotopic and mineralogical investigations of sub$\mu \mathrm{m}$ grains have been performed using a combination of SIMS, transmission electron microscopy (TEM) and focused ion beam (FIB) techniques. Two instruments have been developed to study even smaller grains $(\sim 50 \mathrm{~nm})$ and measure isotopes and elements of lower abundances than those in previous studies.
\end{abstract}

Please cite this article as: S. Amari, Recent Progress in Presolar Grain Studies, Mass Spectrom (Tokyo) 2014; 3(4): S0042; DOI: 10.5702/massspectrometry.S0042

Keywords: presolar grains, isotopic anomalies, nucleosynthesis, meteorites, secondary ion mass spectrometry

(Received August 25, 2014; Accepted November 7, 2014)

\section{INTRODUCTION}

Our solar system formed from a molecular cloud that contained dust and gas expelled from dying stars 4.6 billion years ago. Until the late 1960s it was believed that the solar system was isotopically homogeneous because all dust grains in the molecular cloud were evaporated and material in the molecular cloud was well mixed during the formation. However, when Black and Pepin ${ }^{1)}$ analyzed Ne in primitive meteorites, they found a ${ }^{22} \mathrm{Ne}$-rich component contained in the meteorites $\left({ }^{20} \mathrm{Ne} /{ }^{22} \mathrm{Ne}<3.4,{ }^{20} \mathrm{Ne} /{ }^{22} \mathrm{Ne}_{\text {Air }}=9.8\right)$. The enrichment was so large that it could not be explained by the processes occurring in the solar system and its stellar origin was proposed: meteorites contain a small amount of stardust that survived the solar system formation and subsequent processes in meteorite parent bodies. ${ }^{2)}$ Isotopically anomalous $\mathrm{Kr}$ and $\mathrm{Xe}$ components were also detected in meteorite residues processed by $\mathrm{HF}-\mathrm{HCl}^{3,4)} \mathrm{Kr}-\mathrm{S}$, for $s$-process (slow neutron capture process) $\mathrm{Kr}$, and $\mathrm{Xe}-\mathrm{S}$, for $s$-process $\mathrm{Xe}$, are enriched in even-numbered isotopes relative to the solar ratios. $\mathrm{Xe}-\mathrm{HL}$ is enriched in light, $p$ process (proton capture process) only isotopes 124 and 126 , and heavy, $r$-process (rapid neutron capture process) only isotopes 134 and $136 .{ }^{3)}$ Since the main $s$-process takes place in asymptotic giant branch (AGB) stars and the $p$ - and $r$-processes are believed to take place in supernovae, these anomalies reinforced the idea that stardust was hidden in meteorites.

The identification and isolation of the carrier minerals that contained these anomalous noble gases was performed by Edward Anders, Roy S. Lewis and their colleagues at
The University of Chicago. ${ }^{5-8)}$ A historical account has been described by Anders. ${ }^{9)}$ They dissolved more than 99\% of meteorites they started with during the separation. Diamond was the first mineral type that was isolated in meteorites in $1987,{ }^{5)}$ followed by $\mathrm{SiC}^{6,10)}$ and graphite. ${ }^{11)}$ Interestingly, they all are carbonaceous and quite resistant to chemicals, and that was the reason why they could be concentrated using chemicals. These grains also show isotopic anomalies in elements other than noble gases, proving their stellar origin. These grains are now called presolar grains, which are stardust that formed in stellar outflows or ejecta, remained mostly intact throughout their journey in space, and were eventually incorporated into meteorites.

Mineral types of presolar grains include diamond, ${ }^{5)}$ $\mathrm{SiC},{ }^{6,10)}$ graphite, ${ }^{11)} \mathrm{Si}_{3} \mathrm{~N}_{4}{ }^{12)}$ oxides, ${ }^{13-15)}$ silicates ${ }^{16-19)}$ and refractory carbides inside $\mathrm{SiC}^{20)}$ and graphite. ${ }^{21)}$ Their abundances range from a few ppb (nanograms/gram) to a few hundred ppm (micrograms/gram) relative to bulk samples of undifferentiated chondrites ${ }^{7,22)}$ (Table 1).

Before presolar grains were discovered in meteorites, spectroscopic observations of stars were the only available observations of isotopic ratios in stars. The data obtained this way often have large uncertainties. Presolar grains, on the other hand, can be studied in the laboratory using the state-of-the-art instruments with much higher precision. Studies of presolar grains have yielded a wealth of information about nucleosynthesis in stars, mixing in stellar ejecta, and Galactic chemical evolution (the temporal variation of isotopic and elemental abundances in the Galaxy). There are many aspects of presolar grain studies, which are discussed in detail in review papers. ${ }^{29-32)}$ In this paper we will focus on recent progress that has been made in presolar grain studies

Correspondence to: Sachiko Amari, McDonnell Center for the Space Sciences and the Physics Department, Washington University, One Brookings Drive, Saint Louis Missouri 63130-4899, USA, e-mail: sa@physics.wustl.edu

Abbreviations: AGB, asymptotic giant branch; FIB, focused ion beam; RIMS, resonance ionization mass spectrometry; SIMS, secondary ion mass spectrometry; TEM, transmission electron microscopy; TIMS, thermal ionization mass spectrometry; TOF, time-of-flight 
Table 1. Presolar grains in meteorites.

\begin{tabular}{|c|c|c|c|c|}
\hline Mineral & Year of discovery & Size & Abundance* & References \\
\hline Diamond & 1987 & Average: $30 \mathrm{~nm}$ & $\sim 500 \mathrm{ppm}$ & $5,7,23)$ \\
\hline $\mathrm{SiC}$ & $1987 / 1988$ & $\begin{array}{c}\text { Mostly }<1 \mu \mathrm{m}, \\
\text { up to } 50 \mu \mathrm{m} \text { (rare) }\end{array}$ & $5.9 \mathrm{ppm}$ & 7) \\
\hline Graphite & 1990 & $>1 \mu \mathrm{m}$ & $0.88 \mathrm{ppm}$ & $7,24)$ \\
\hline $\begin{array}{l}\text { Refractory carbides } \\
\text { (as subgrains) }\end{array}$ & 1991 & Up to a few hundred nm & $\begin{array}{c}\text { (Present in host graphite/ } \\
\text { SiC grains) }\end{array}$ & $21,25,26)$ \\
\hline Oxides & 1994 & Mostly $<1 \mu \mathrm{m}$, up to a few $\mu \mathrm{m}$ & $1-3 \mathrm{ppm}^{* *}$ & $13-15,22,27)$ \\
\hline $\mathrm{Si}_{3} \mathrm{~N}_{4}$ & 1995 & Up to a few $\mu \mathrm{m}$ & A few ppb & 12) \\
\hline Silicates & 2004 & $<1 \mu \mathrm{m}$ & $29-57 \mathrm{ppm}^{* *}$ & $17-19,22,27)$ \\
\hline
\end{tabular}

* These numbers are relative to bulk samples of undifferentiated chondrites. The abundances of diamond, SiC and graphite are those in the Murchison meteorite (CM2).

** 100-200 ppm of silicates+oxides are a matrix-normalized abundance. ${ }^{22)}$ In CO and CR chondrites where many of the abundance data have been obtained, the matrix comprise $30 \mathrm{vol} \%$ and $30-50$ vol\% of meteorites, respectively. ${ }^{28)}$ Taking 30 vol\% for the average and assuming that the matrix and the rest of the meteorites have the same density, the abundance of silicates+oxides relative to a bulk meteorite would be $30-60$ ppm. The average ratio of silicates to oxides in CR chondrites is $22 .^{27)}$

during the last $10-15$ years.

\section{IDENTIFICATION OF PRESOLAR SILICATE GRAINS}

Presolar $\mathrm{SiC}$ and, to lesser extent, graphite have been extensively studied using noble gas mass spectrometry, thermal ionization mass spectrometry (TIMS), and SIMS. There are two ways to analyze presolar grains. One way is to analyze a large number of grains at the same time (bulk analysis) and the other way is to analyze grains one by one. Most of the single grain data were obtained using SIMS. Since each presolar grain might have formed in a different star, single grain analysis would be best suited for presolar grains. A drawback is that these analyses require relatively large grains $(>1 \mu \mathrm{m})$ and high elemental abundances in the grains. Isotopic ratios of $\mathrm{C}, \mathrm{N}, \mathrm{Si}, \mathrm{Mg}-\mathrm{Al}, \mathrm{Ca}$ and $\mathrm{Ti}$ in $\mathrm{SiC}$ grains analyzed with SIMS ${ }^{33)}$ and those of $\mathrm{Kr}$, Xe, Sr, Ba with noble gas mass spectrometry ${ }^{34-36)}$ and TIMS $^{37-39)}$ indicate that most $\mathrm{SiC}$ grains ( $93 \%$ of the total $\mathrm{SiC}$ grains) formed in AGB stars. ${ }^{40-42)}$

Carbon-rich stars produce carbonaceous grains while O-rich stars produce oxides and silicates (silicates can still form in C-rich conditions but mainly form in O-rich conditions). Studies of presolar oxides and silicates would give us much information of O-rich stars. The difficulty is, however, how to find presolar oxides and silicates in meteorites.

Carbonaceous grains are chemically resistant thus it is possible to use chemicals, such as hydrofluoric acid (HF) and dichromate solution $\left(\mathrm{Cr}_{2} \mathrm{O}_{7}{ }^{2-}\right)$, to remove the other phases to concentrate the carbonaceous grains. Since oxides are also chemically resistant, we could concentrate them together with these carbonaceous grains. The difference between C-rich and oxide grains is that most of the oxide grains in meteorites formed in the solar system. We needed a method to efficiently locate presolar oxides from the sea of solar oxide grains. Ion Imaging techniques were developed to find rare types of grains in low abundances from overwhelmingly abundant normal grains. ${ }^{13)}$ Isotopic images of a $100 \times 100 \mu \mathrm{m}$ area, where oxide grains are dispersed, are taken using a defocused beam. Grains are defined from a grain-definition algorithm and isotopic ratios of the grains are calculated from the images. This method enables us to quickly locate presolar oxides, which comprise much less than $1 \%$ of the oxide grains in meteorites.

The search for presolar silicates was much more labor intensive. Most of the bulk meteorites were dissolved to concentrate oxide grains to search for presolar oxides. This method was out of question in the search for presolar silicates. Silicates are rock-forming minerals and meteorites consist mostly of silicates. If we dissolved solar silicates, presolar silicates would have been equally destroyed. Therefore, either meteorite thin sections or dispersed meteorite materials were examined. Ion imaging techniques had been also applied to search for presolar silicate grains using the CAMECA IMS-3f at Washington University in Saint Louis in the late 1990s with no avail. ${ }^{43)}$ In hindsight, the spatial resolution of the CAMECA IMS-3f was not enough to locate presolar silicates because the diameter of a $\mathrm{Cs}^{+}$primary beam of the CAMECA IMS-3f was a few $\mu \mathrm{m}$. In addition, the sensitivity was not high enough to obtain high signal to noise ratios. We had to wait until a new type of SIMS was constructed and delivered.

The CAMECA NanoSIMS was developed in the late 1990s and the first NanoSIMS was delivered at Washington University in December 2000. A Cs ${ }^{+}$primary beam diameter can be as small as $50 \mathrm{~nm}$ and this made it possible to find isotopic anomalies in much smaller grains/areas in meteorites than previously possible. ${ }^{44)}$ The NanoSIMS is equipped with 4 moveable and one fixed electron multipliers that can simultaneously detect five ions up to mass 30 . Together with the ion optics, the sensitivity of the NanoSIMS is much higher than that of the CAMECA IMS-3f. Presolar silicate grains were first found in interplanetary dust particles ${ }^{16)}$ followed by their discovery in meteorites. ${ }^{17-19)}$ Silicate grains have been located in various types of meteorites that had experienced the least amount of aqueous alteration. ${ }^{22)}$ Their $\mathrm{O}$ isotopic distribution is similar to that of oxide grains (see Fig. 14 by Zinner ${ }^{32)}$ for the $\mathrm{O}$ isotopic distribution of oxides): grains are classified into four groups according to their $\mathrm{O}$ isotopic ratios ${ }^{45)}$ (Fig. 1) . Group 1 grains show ${ }^{17} \mathrm{O}$ excesses and close-to-solar ${ }^{18} \mathrm{O} /{ }^{16} \mathrm{O}$ ratios. Group 2 grains have ${ }^{17} \mathrm{O}$ excesses and ${ }^{18} \mathrm{O}$ deficits and the latter are more pronounced than the former. Group 3 grains show ${ }^{16} \mathrm{O}$ excesses whereas Group 4 grains show ${ }^{17} \mathrm{O}$ and ${ }^{18} \mathrm{O}$ excesses. Grains of Groups 1,2 and 3 formed in red giants or AGB stars ${ }^{45)}$ and those of Group 4 most likely formed in supernovae. ${ }^{46,47)}$ With the identification of presolar silicate grains, major types of 


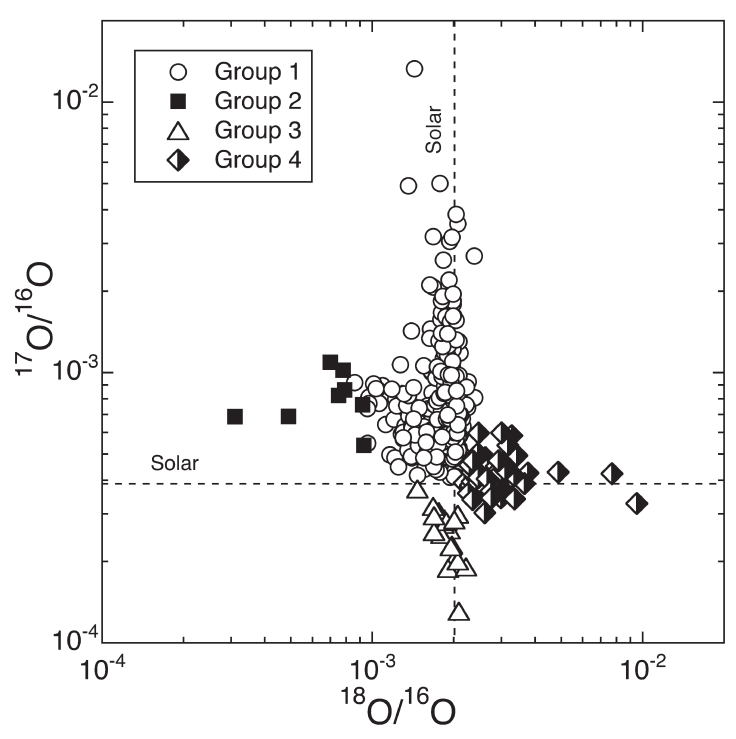

Fig. 1. Oxygen isotopic ratios of presolar silicate grains. Data are from the Presolar Grain Database ${ }^{48)}$ (http://presolar.wustl. edu/PGD/Presolar_Grain_Database.html). The grains are classified into four groups based on their $\mathrm{O}$ isotopic ratios (see the text). The dotted lines indicate the solar ratios.

presolar grains formed in both C-rich and O-rich stars have been identified in meteorites.

\section{STUDIES BY THE COMBINATION OF DIFFER- ENT TYPES OF INSTRUMENT}

Isotopic analyses of presolar grains are essential for presolar grain studies. To establish the presolar origin of grains, isotopic anomalies in at least one element have to be present. NanoSIMS analyses consume less material than previous analyses by the CAMECA IMS-3f, thus leaving more material for subsequent measurements by other types of instrument. The crystal structure and presence of subgrains inside host carbonaceous grains have been examined using TEM. ${ }^{21,25,26,49,50)}$ The presence of subgrains and their elemental compositions in graphite grains revealed formation conditions of the subgrains and their host graphite grains. ${ }^{25)}$ Isotopic analyses of subgrains in thin $(\sim 70 \mathrm{~nm})$ slices of graphite grains ${ }^{51,52)}$ could be also performed. Titanium isotopic ratios of $\mathrm{TiC}$ subgrains $(<\sim 500 \mathrm{~nm})$ in a graphite grain of supernova origin agreed with those measured in the host graphite grain and were consistent with their supernova origin. ${ }^{51)}$ Silicon carbide subgrains with ${ }^{29,30} \mathrm{Si}$ excesses (up to 2 times solar ${ }^{29} \mathrm{Si} /{ }^{28} \mathrm{Si}$ and ${ }^{30} \mathrm{Si} /{ }^{28} \mathrm{Si}$ ratios) were identified in two graphite grains. ${ }^{52)}$ Such high excesses could only be explained by C-burning and/or Ne-burning in massive stars $\left(>8 \mathrm{M}_{\text {sun }}\right)$. Croat et al ${ }^{52)}$ suggested that these $\mathrm{SiC}$ grains were later incorporated into the graphite grains, either in supernova ejecta or in the colliding winds of Wolf-Rayet binaries.

TEM, in combination with the FIB technique, has been widely used to examine presolar silicate grains. Normal silicate grains usually surround presolar silicate grains. Even with a small primary beam with the NanoSIMS, signals from nearby grains cannot be totally excluded. Oxygen isotopic ratios, which are used to locate presolar silicate grains, are usually vastly different from the solar ratios thus the interference from nearby solar grains has little effect on locating these grains. However, other isotopic ratios, such as those of $\mathrm{Mg}$ and $\mathrm{Si}$, of presolar silicates are usually not so different from the solar ratios. To precisely determine isotopic ratios of these other elements, it is necessary to remove surrounding grains. Nguyen and Messenger ${ }^{53)}$ placed a Pt cap to protect a silicate grain of interest and milled surrounding grains to analyze $\mathrm{Mg}, \mathrm{Si}$ and $\mathrm{Fe}$ isotopic ratios (see Fig. 1 by Nguyen and Messenger ${ }^{53)}$ ). From isotopic analyses of the 11 grains measured this way, they estimated $\sim 12 \%$ and $1 \%$ of presolar silicates have supernova and nova origins, and the rest originated from red giants and AGB stars.

The structures of presolar grains reflect condensation conditions of these grains as well as subsequent processing histories. Observations using the ISO SWS (Infrared Space Observatory Short-Wavelength Spectrometer) indicates that a widespread presence of crystalline silicates in the envelopes of evolved stars. ${ }^{54)}$ It is assumed that a portion of freshly condensed amorphous silicate grains is converted into crystalline silicates by thermal annealing in the circumstellar shell. ${ }^{55)}$ TEM examinations have been carried out for both presolar oxide and silicate grains. Corundum $\left(\mathrm{Al}_{2} \mathrm{O}_{3}\right),{ }^{56)}$ hibonite $\left(\mathrm{CaAl}_{12} \mathrm{O}_{19}\right),{ }^{27,57)}$ and spinel $\left(\mathrm{MgAl}_{2} \mathrm{O}_{4}\right.$ and $\mathrm{Fe}$ - and $\mathrm{Cr}$-rich with varied amounts of $\mathrm{Mg}$ and $\mathrm{Al})^{58)}$ have been investigated using FIB and TEM techniques. Except for one amorphous $\mathrm{Al}_{2} \mathrm{O}_{3}$ grain and an assemblage of three $\mathrm{Fe}-\mathrm{Cr}$-rich crystalline grains, they are single crystal grains. One highly crystalline orthopyroxene $\left(\mathrm{MgSiO}_{3}\right)$ grain with a high $\mathrm{Al}$ content (1.8 \pm 0.5 at.\%), and an amorphous $\mathrm{Ca}-\mathrm{Si}$ rich grain, with hibonite nanocrystallites (< $20 \mathrm{~nm}$ ) embedded in one region, have been found in the Acfer 094 meteorite. ${ }^{59)}$ Vollumer et al. ${ }^{59)}$ suggested that the complex assemblage of the latter indicated the fast cooling and changing chemical environments where the grain had formed.

\section{FUTURE DIRECTIONS}

Presolar grain studies are a relatively new field, starting in 1987. ${ }^{5)}$ Progress has been made in many aspects such as nucleosynthesis in AGB stars and supernovae, mixing in supernova and nova ejecta, and the Galactic chemical evolution. There are still a few aspects yet to be explored.

There are most likely other presolar-grain minerals that have not been isolated/identified in meteorites. Besides the major mineral types of presolar grains identified to date [diamond, $\mathrm{SiC}$, graphite, oxides, silicates, $\mathrm{Si}_{3} \mathrm{~N}_{4}$, refractory carbides (TiC, Mo- and Zr-rich carbides) as subgrains], condensation calculations predict sulfides would form in sufficiently reducing stellar environments. ${ }^{60)}$ Sulfides are dissolved during an alternate treatment with $\mathrm{HF}-\mathrm{HCl}$ and $\mathrm{HCl}$ in the chemical separation procedure. Thus, some other methods to remove other phases are needed to concentrate presolar sulfides, if they exist. How to effectively concentrate minerals of interest pauses a challenge to identify new phases. Besides the difficulty of concentrating the new phases, their low abundances, probably on the order of a few ppm (micrograms/gram) or less relative to bulk meteorites, make the task even more challenging.

Another aspect is to analyze isotopic ratios of heavy elements in single grains. Isotopic ratios of heavy elements were analyzed mostly on bulk samples. ${ }^{34-39,61)}$ Although high precision data were obtained, a large number of grains were analyzed at the same time and it was hard to correlate 
isotopic ratios of multiple elements even if aliquots of the same fraction were used for analyses. SIMS, used to acquire isotopic ratios of single grains, has been mostly used to analyze isotopic ratios of light to intermediate elements such as $\mathrm{C}, \mathrm{N}, \mathrm{O}, \mathrm{Mg}, \mathrm{Si}, \mathrm{Ca}, \mathrm{Ti}, \mathrm{Fe}$ and $\mathrm{Ni}^{24,33,62)}$ It is very hard to analyze isotopic ratios of heavy elements using SIMS because extremely high mass resolving power $(M / \Delta M)$ is necessary to separate interferences in the mass region of heavy elements: to separate ${ }^{96} \mathrm{Mo}$ and ${ }^{96} \mathrm{Zr}$, a $\mathrm{M} / \Delta \mathrm{M}$ of 22,120 is required and this would considerably reduce sensitivity. In addition, abundances of elements heavier than $\mathrm{Fe}$ are quite low in nature. ${ }^{63)}$

Isotopic ratios of heavy elements are important to decipher nucleosynthesis in stars. The $s$-process takes place in AGB stars and massive stars $\left(>8 \mathrm{M}_{\text {sun }}\right)$ and isotopic yields of elements affected by $s$-process branching are good indicators of neutron density, neutron exposure (i.e., the total number of neutrons) and temperature. Thus these pieces of information would provide us with nucleosynthetic conditions in the stars. Other processes that synthesize heavy elements are the $p$-process and $r$-process and they are thought to take place in supernovae. The $p$-process is a photo-disintegration processes $[(\gamma, \mathrm{n}),(\gamma, \mathrm{p})$ and $(\gamma, \alpha)]$ of pre-existing neutron-rich nuclei under high temperature $\left(T>\sim 2 \times 10^{9} \mathrm{~K}\right)$. The $r$-process is thought to occur in neutrino-driven winds from the neutron star of core-collapse supernovae. ${ }^{64)}$ Other candidate locations include the "hot bubble (high entropy)" region, which is believed to appear just after the creation of a newlyborn proto-neutron star in the core-collapse supernova explosion, neutron star-neutron star binaries, and neutron star-black hole binaries. These processes can be better understood when isotopic ratios of heavy elements in supernova presolar grains are analyzed with high precision.

Isotopic ratios of heavy elements in a few graphite and $\mathrm{SiC}$ grains have been analyzed using resonance ionization mass spectrometry (RIMS). Atoms, desorbed from samples with a laser, are resonantly ionized with several tuned lasers. Thus only elements of interest are ionized. These ions are analyzed by a time-of-flight (TOF) mass spectrometer. An advantage is that RIMS has higher ionization yields than SIMS. Useful yields, defined as the number of detected ions to the number of sputtered atoms, are a few \% (up to $25 \%$ ) in the former, while they are less than $1 \%$ in the latter.

Isotopic ratios of Mo and $\mathrm{Zr},{ }^{65-69)} \mathrm{Sr}^{70,71)} \mathrm{Ba}^{71,72)}$ and $\mathrm{Ru}^{73)}$ in single $\mathrm{SiC}$ and graphite grains have been analyzed using the RIMS, CHARISMA instrument (Chicago-Argonne Resonant Ionization Spectrometer for Mass Analysis), at Argonne National Laboratory, USA. ${ }^{74)}$ These isotopic analyses of mainstream $\mathrm{SiC}$ grains, which comprise $\sim 93 \%$ of the total $\mathrm{SiC}$ grains, have shown signatures of $s$-process in low-mass (1-3 $\mathrm{M}_{\text {sun }}$ ) AGB stars of close-to-solar metallicity (metallicity indicates the mass fraction of elements heavier than $\mathrm{He}$ ). Silicon carbide grains of Type X, comprising $1 \%$ of total $\mathrm{SiC}$, are considered to originate from supernovae. Molybdenum and $\mathrm{Zr}$ isotopic ratios of $\mathrm{X}$ grains ${ }^{65,71)}$ show signature of neutron burst, or mini $r$-process that takes place in the He-rich zone during the passage of the shock wave from the core. ${ }^{75)}$ In neutron burst, neutron exposure $\tau$ is estimated to be $0.07-0.08 \mathrm{mbarn}^{-1}$ at $T_{\max }=10^{9} \mathrm{~K}^{75)}$ and most neutrons are captured by seed nuclei, resulting in a few neutrons captured per nucleus. In the $r$-process, neutron exposure $\tau$ is calculated to be $\sim 1.2 \times 10^{10} \mathrm{mbarn}^{-1}$ at $T=10^{9} \mathrm{~K}$ (Meyer, personal communication), but most of the neutrons are lost via the $(\gamma, \mathrm{n})$ reaction and $\sim 100$ neutrons per nucleus are captured in the end.

A few high-density graphite grains have shown signatures of $s$-process in AGB stars. ${ }^{69)}$ Ruthenium isotopic analysis of $\mathrm{SiC}$ grains ${ }^{73)}$ is particularly interesting: Savina et al. ${ }^{73)}$ have found a ${ }^{99} \mathrm{Ru}$ anomaly that is explained by the in situ decay of ${ }^{99} \mathrm{Tc}\left(T_{1 / 2}=213,000 \mathrm{a}\right)$ in $\mathrm{SiC}$ grains. Technetium has been spectroscopically observed in AGB stars. ${ }^{76)}$ Since there are no stable Tc isotopes, the observation has been taken as evidence that nucleosynthesis is taking place in stars. Both the grain analysis and the observation of stars agreed with each other and proved nucleosynthesis takes place in stars.

Although CHARISMA has produced new insights into nucleosynthesis in stars from these isotopic measurements, the grains measured in the above analyses were at least a few $\mu \mathrm{m}$ in size. The effort has been made to analyze isotopic and elemental abundances with much higher precision and in much smaller (down to a nanometer range) grains and areas. Two instruments have been developed. CHILI (The Chicago Instrument for Laser Ionization), under construction at The University of Chicago, USA, is a newer version of RIMS. ${ }^{77)}$ The goal is to have a useful yield of $\sim 40 \%$. A liquid metal ion gun is used to analyze $\sim 10 \mathrm{~nm}$ scale samples. Six tunable lasers will simultaneously ionize up to three elements.

Another instrument, LIMAS (Laser Ionization Mass Nanoscope), is located at Hokkaido University, Japan. The prototype was developed at Osaka University in collaboration with Hokkaido University, Kyushu University, Hitachi High-Tech Science Corporation, and JEOL in Japan. ${ }^{78)}$ A liquid metal $\mathrm{Ga}$ ion source is used for a primary beam. Atoms are desorbed and are non-resonantly ionized by a femtosecond laser, which is capable of yielding very high-power densities. The femtosecond laser is focused at a point $100 \mu \mathrm{m}$ above the surface of a sample. ${ }^{79)}$ High-mass resolution power is needed to eliminate interferences after non-resonant ionization of samples. A multi-turn TOF mass spectrometer, MULTUM II, can achieve mass resolving power of 250,000 when ions are focused in both space and time. ${ }^{80)}$ The prototype is at Osaka University and is in the process of modification to optimize the performance. The main focus would be to optimize post ionization efficiencies and tuning of the MULTUM II to minimize the loss of ions. ${ }^{81)}$

Although it will take some time before these instruments are in full operation, we expect isotopic and elemental abundances of extra-terrestrial materials will be analyzed in unprecedented precisions. In the past, improvements of instrument always bring some new insights and it remains to be seen what kind of new information these instruments will reveal.

\section{Acknowledgements}

The presentation of this work at The 62nd Annual Conference on Mass Spectrometry in May 14-16, 2014 and the presentation at the Future Research Initiative Group Project Support Program Symposium on May 17, 2014 were supported by the project entitled 'On-Site Mass Spectrometry Based on the Multi-Turn Time-of-Flight Mass Spectrometer "MULTUM" (PI: Michisato Toyoda)' in The Support Program for Osaka University Future Research Initiative 
Groups. The author enjoyed simultaneous discussion during the conference and the symposium and the laboratory tour at Osaka University on May 17, where new and innovative instruments were under development. The author is grateful for discussion with Bradley S. Meyer about nucleosynthesis, and for the constructive comments from the editor and the two anonymous reviewers, which have improved the manuscript.

\section{REFERENCES}

1) D. C. Black, R. O. Pepin. Trapped neon in meteorites. II. Earth Planet. Sci. Lett. 6: 395-405, 1969.

2) D. D. Clayton. Na-22, Ne-E, extinct radioactive anomalies and unsupported Ar-40. Nature 257: 36-37, 1975.

3) R. S. Lewis, B. Srinivasan, E. Anders. Host phase of a strange xenon component in Allende. Science 190: 1251-1262, 1975.

4) B. Srinivasan, E. Anders. Noble gases in the Murchison meteorite: Possible relics of $s$-process nucleosynthesis. Science 201: 51-56, 1978

5) R. S. Lewis, M. Tang, J. F. Wacker, E. Anders, E. Steel. Interstellar diamonds in meteorites. Nature 326: 160-162, 1987.

6) M. Tang, E. Anders. Isotopic anomalies of $\mathrm{Ne}, \mathrm{Xe}$, and $\mathrm{C}$ in meteorites. II. Interstellar diamond and $\mathrm{SiC}$ : Carriers of exotic noble gases. Geochim. Cosmochim. Acta 52: 1235-1244, 1988.

7) S. Amari, R. S. Lewis, E. Anders. Interstellar grains in meteorites: I. Isolation of $\mathrm{SiC}$, graphite, and diamond; Size distributions of SiC and graphite. Geochim. Cosmochim. Acta 58: 459-470, 1994.

8) G. R. Huss, R. S. Lewis. Presolar diamond, SiC, and graphite in primitive chondrites: Abundances as a function of meteorite class and petrologic type. Geochim. Cosmochim. Acta 59: 115-160, 1995.

9) E. Anders. Circumstellar material in meteorites: Noble gases, carbon and nitrogen. In Meteorites and the Early Solar System (Ed: J. F. Kerridge, M. S. Matthews), University of Arizona Press, Tucson, 1988, pp. 927-955.

10) T. Bernatowicz, G. Fraundorf, M. Tang, E. Anders, B. Wopenka, E. Zinner, P. Fraundorf. Evidence for interstellar $\mathrm{SiC}$ in the Murray carbonaceous meteorite. Nature 330: 728-730, 1987.

11) S. Amari, E. Anders, A. Virag, E. Zinner. Interstellar graphite in meteorites. Nature 345: 238-240, 1990.

12) L. R. Nittler, P. Hoppe, C. M. O’D Alexander, S. Amari, P. Eberhardt, X. Gao, R. S. Lewis, R. Strebel, R. M. Walker, E. Zinner. Silicon nitride from supernovae. Astrophys. J. 453: L25-L28, 1995.

13) L. R. Nittler, C. M. O'D Alexander, X. Gao, R. M. Walker, E. K. Zinner. Interstellar oxide grains from the Tieschitz ordinary chondrite. Nature 370: 443-446, 1994.

14) I. D. Hutcheon, G. R. Huss, A. J. Fahey, G. J. Wasserburg. Extreme ${ }^{26} \mathrm{Mg}$ and ${ }^{17} \mathrm{O}$ enrichments in an Orgueil corundum: Identification of a presolar oxide grain. Astrophys. J. 425: L97-L100, 1994.

15) G. R. Huss, A. J. Fahey, R. Gallino, G. J. Wasserburg. Oxygen isotopes in circumstellar $\mathrm{Al}_{2} \mathrm{O}_{3}$ grains from meteorites and stellar nucleosynthesis. Astrophys. J. 430: L81-L84, 1994.

16) S. Messenger, L. P. Keller, F. J. Stadermann, R. M. Walker, E. Zinner. Samples of stars beyond the solar system: Silicate grains in interplanetary dust. Science 300: 105-108, 2003.

17) A. N. Nguyen, E. Zinner. Discovery of ancient silicate stardust in a meteorite. Science 303: 1496-1499, 2004.

18) K. Nagashima, A. N. Krot, H. Yurimoto. Stardust silicates from primitive meteorites. Nature 428: 921-924, 2004

19) S. Mostefaoui, P. Hoppe. Discovery of abundant in situ silicate and spinel grains from red giant stars in a primitive meteorite. Astrophys. J. 613: L149-L152, 2004.

20) T. J. Bernatowicz, S. Amari, R. S. Lewis. TEM studies of a circumstellar rock. Lunar Planet. Sci. 23: 91-92, 1992.
21) T. J. Bernatowicz, S. Amari, E. K. Zinner, R. S. Lewis. Interstellar grains within interstellar grains. Astrophys. J. 373: L73-L76, 1991.

22) C. Floss, F. Stadermann. Presolar silicate and oxide abundances and compositions in the ungrouped carbonaceous chondrite Adelaide and the K chondrite Karangari: The effects of secondary processing. Meteorit. Planet. Sci. 47: 992-1009, 2012.

23) T. L. Daulton, D. D. Eisenhour, T. J. Bernatowicz, R. S. Lewis, P. R. Buseck. Genesis of presolar diamonds: Comparative highresolution transmission electron microscopy study of meteoritic and terrestrial nano-diamonds. Geochim. Cosmochim. Acta 60: 4853-4872, 1996

24) S. Amari, E. Zinner, R. Gallino. Presolar graphite from the Murchison meteorite: An isotopic study. Geochim. Cosmochim. Acta 133: 479-522, 2014

25) T. J. Bernatowicz, R. Cowsik, P. C. Gibbons, K. Lodders, B. Fegley Jr., S. Amari, R. S. Lewis. Constraints on stellar grain formation from presolar graphite in the Murchison meteorite. Astrophys. J. 472: 760-782, 1996.

26) T. K. Croat, T. Bernatowicz, S. Amari, S. Messenger, F. J. Stader mann. Structural, chemical, and isotopic microanalytical investigations of graphite from supernovae. Geochim. Cosmochim. Acta 67: 4705-4725, 2003.

27) J. Leitner, C. Vollmer, P. Hoppe, J. Zipfel. Characterization of presolar material in the CR chondrite Northwest Africa 852. Astrophys. J. 745: 38, 2012.

28) E. R. D. Scott, S. G. Love, A. N. Krot. Formation of chondrules and chondrites in the protoplanetary nebula. In Chondrules and the Protoplanetary Disk (Ed: R. H. Hewins, R. H. Jones, E. R. D. Scott), Cambridge University Press, Cambridge, 1996, pp. 87-96.

29) E. Zinner. Stellar nucleosynthesis and the isotopic composition of presolar grains from primitive meteorites. Annu. Rev. Earth Planet. Sci. 26: 147-188, 1998.

30) D. D. Clayton, L. R. Nittler. Astrophysics with presolar stardust. Annu. Rev. Astron. Astrophys. 42: 39-78, 2004.

31) K. Lodders, S. Amari. Presolar grains from meteorites: Remnants from the early times of the solar system. Chem. Erde 65: 93-166, 2005.

32) E. Zinner. Presolar grains. In Treatise on Geochemistry (Ed: H D. Holland, K. K. Turekian, A. M. Davis), Elsevier, Oxford, 2013, pp. 181-213.

33) P. Hoppe, S. Amari, E. Zinner, T. Ireland, R. S. Lewis. Carbon, nitrogen, magnesium, silicon and titanium isotopic compositions of single interstellar silicon carbide grains from the Murchison carbonaceous chondrite. Astrophys. J. 430: 870-890, 1994.

34) U. Ott, F. Begemann, J. Yang, S. Epstein. s-Process $\mathrm{Kr}$ of variable isotopic composition in the Murchison meteorite. Nature 332: 700-702, 1988 .

35) R. S. Lewis, S. Amari, E. Anders. Meteoritic silicon carbide: Pristine material from carbon stars. Nature 348: 293-298, 1990.

36) R. S. Lewis, S. Amari, E. Anders. Interstellar grains in meteorites: II. SiC and its noble gases. Geochim. Cosmochim. Acta 58: 471-494, 1994.

37) U. Ott, F. Begemann. Discovery of s-process barium in the Murchison meteorite. Astrophys. J. 353: L57-L60, 1990.

38) C. A. Prombo, F. A. Podosek, S. Amari, R. S. Lewis. s-Process Ba isotopic compositions in presolar $\mathrm{SiC}$ from the Murchison meteorite. Astrophys. J. 410: 393-399, 1993.

39) F. A. Podosek, C. A. Prombo, S. Amari, R. S. Lewis. s-Process Sr isotopic compositions in presolar $\mathrm{SiC}$ from the Murchison meteorite. Astrophys. J. 605: 960-965, 2004.

40) R. Gallino, M. Busso, G. Picchio, C. M. Raiteri. On the astrophysical interpretation of isotope anomalies in meteoritic $\mathrm{SiC}$ grains. Nature 348: 298-302, 1990.

41) R. Gallino, C. M. Raiteri, M. Busso. Carbon stars and isotopic Ba anomalies in meteoritic SiC grains. Astrophys. J. 410: 400-411 1993.

42) R. Gallino, C. M. Raiteri, M. Busso, F. Matteucci. The puzzle of silicon, titanium and magnesium anomalies in meteoritic silicon 
carbide grains. Astrophys. J. 430: 858-869, 1994.

43) S. Messenger, T. J. Bernatowicz. Search for presolar silicates in Acfer 094. Meteorit. Planet. Sci. 35: A109, 2000.

44) F. J. Stadermann, R. M. Walker, E. Zinner. NanoSIMS: The next generation ion probe for the microanalysis of extraterrestrial material. Meteorit. Planet. Sci. 34: A111-A112, 1999.

45) L. R. Nittler. C. M. O’D Alexander, X. Gao, R. M. Walker, E. Zinner. Stellar sapphires: The properties and origins of presolar $\mathrm{Al}_{2} \mathrm{O}_{3}$ in meteorites. Astrophys. J. 483: 475-495, 1997.

46) B.-G. Choi, G. R. Huss, G. J. Wasserburg, R. Gallino. Presolar corundum and spinel in ordinary chondrites: Origins from AGB stars and a supernova. Science 282: 1284-1289, 1998.

47) L. R. Nittler, C. M. O'D Alexander, R. Gallino, P. Hoppe, A. N. Nguyen, F. J. Stadermann, E. K. Zinner. Aluminum-, calciumand titanium-rich oxide stardust in ordinary chondrite meteorites. Astrophys. J. 682: 1450-1478, 2008.

48) K. M. Hynes, F. Gyngard. The presolar grain database: http:// presolar.wustl.edu/ pgd. Lunar Planet. Sci. 40: Abstract \#1198, 2009.

49) T. K. Croat, F. J. Stadermann, T. J. Bernatowicz. Presolar graphite from AGB stars: Microstructure and s-process enrichment. Astrophys. J. 631: 976-987, 2005.

50) K. M. Hynes, T. K. Croat, S. Amari, A. F. Mertz, T. J. Bernatowicz. Structual and isotopic microanalysis of presolar $\mathrm{SiC}$ from supernovae. Meteorit. Planet. Sci. 45: 596-614, 2010.

51) F. J. Stadermann, T. K. Croat, T. J. Bernatowicz, S. Amari, S. Messenger, R. M. Walker, E. Zinner. Supernova graphite in the NanoSIMS: Carbon, oxygen and titanium isotopic compositions of a spherule and its TiC sub-components. Geochim. Cosmochim. Acta 69: 177-188, 2005.

52) T. K. Croat, F. J. Stadermann, T. J. Bernatowicz. Unusual ${ }^{29,30} \mathrm{Si}-$ rich SiCs of massive star origin found within graphites from the Murchison meteorite. Astron. J. 139: 2159-2169, 2010.

53) A. N. Nguyen, S. Messenger. Resolving the stellar sources of isotopically rare presolar silicate grains through $\mathrm{Mg}$ and $\mathrm{Fe}$ isotopic analyses. Astrophys. J. 784: 149, 2014.

54) L. B. F. M. Waters, F. J. Molster, T. de Jong, D. A. Beintema, C. Waelkens, A. C. A. Boogert, D. R. Boxhoorn, T. de Graauw, S. Drapatz, H. Feuchtgruber, R. Genzel, F. P. Helmich, A. M. Heras, R. Huygen, H. Izumiura, K. Justtanont, D. J. M. Kester, D. Kunze, F. Lahuis, H. J. G. L. M. Lamers, K. J. Leech, C. Loup, D. Lutz, P. W. Morris, S. D. Price, P. R. Roelfsema, A. Salama, S. G. Schaeidt, A. G. G. M. Tielens, N. R. Trams, E. A. Valentijn, B. Vandenbussche, M. E. van den Ancker, E. F. van Dishoeck, H. Van Winckel, P. R. Wesselius, E. T. Young. Mineralogy of oxygen-rich dust shells. Astron. Astrophys. 315: L361-L364, 1996.

55) S. L. Hallenbeck, J. A. Nuth III, P. L. Daukantas. Mid-infrared spectral evolution of amorphous magnesium silicate smokes annealed in vacuum: Comparison to cometary spectra. Icarus 131: 198-209, 1998.

56) R. M. Stroud, L. R. Nittler, C. M. O'D Alexander. Polymorphism in presolar $\mathrm{Al}_{2} \mathrm{O}_{3}$ grains from asymptotic giant branch stars. Science 305: 1455-1457, 2004.

57) T. J. Zega, C. M. O’D Alexander, L. R. Nittler, R. M. Stroud. A transmission electron microscopy study of presolar hibonite. Astrophys. J. 730: 83, 2011.

58) T. J. Zega, L. R. Nittler, F. Gyngard, C. M. O’D Alexander, R. M. Stroud, E. K. Zinner. A transmission electron microscopy study of presolar spinel. Geochim. Cosmochim. Acta 124: 152-169, 2014.

59) C. Vollmer, P. Hoppe, F. E. Brenker. Transmission electron microscopy of Al-rich silicate stardust from asymptotic giant branch stars. Astrophys. J. 769: 61, 2013.

60) K. Lodders, B. Fegley Jr. The origin of circumstellar silicon carbide grains found in meteorites. Meteoritics 30: 661-678, 1995.

61) S. Amari, R. S. Lewis, E. Anders. Interstellar grains in meteorites: III. Graphite and its noble gases. Geochim. Cosmochim Acta 59: 1411-1426, 1995

62) K. K. Marhas, S. Amari, F. Gyngard, E. Zinner, R. Gallino. Iron and nickel isotopic ratios in presolar $\mathrm{SiC}$ grains. Astrophys. J. 689: 622-645, 2008

63) K. Lodders, H. Palme, H.-P. Gail. Abundances of the elements in the solar system. In Landolt-Börnstein, New Series (Ed: J. E. Trümper), Springer-Verlag, Berlin, 2009, pp. 560-630.

64) S. Wanajo, T. Kajino, G. J. Mathews, K. Otsuki. The $r$-process in neutrino-driven winds from nascent, "compact" neutron stars of core-collapse supernovae. Astrophys. J. 554: 578-586, 2001.

65) M. J. Pellin, A. M. Davis, R. S. Lewis, S. Amari, R. N. Clayton. Molybdenum isotopic composition of single silicon carbide grains from supernovae. Lunar Planet. Sci. 30: Abstract \#1969, 1999.

66) M. J. Pellin, A. M. Davis, W. F. Calaway, R. S. Lewis, R. N. Clayton, S. Amari. Zr and Mo isotopic constraints on the origin of unusual types of presolar SiC grains. Lunar Planet. Sci. 31: Abstract $\# 1934,2000$.

67) G. K. Nicolussi, A. M. Davis, M. J. Pellin, R. S. Lewis, R. N. Clayton, S. Amari. $s$-Process zirconium in presolar silicon carbide grains. Science 277: 1281-1283, 1997.

68) G. K. Nicolussi, M. J. Pellin, R. S. Lewis, A. M. Davis, S. Amari, R. N. Clayton. Molybdenum isotopic composition of individual presolar silicon carbide grains from the Murchison meteorite. Geochim. Cosmochim. Acta 62: 1093-1104, 1998.

69) G. K. Nicolussi, M. J. Pellin, R. S. Lewis, A. M. Davis, R. N. Clayton, S. Amari. Zirconium and molybdenum in individual circumstellar graphite grains: New isotopic data on the nucleosynthesis of heavy elements. Astrophys. J. 504: 492-499, 1998.

70) G. K. Nicolussi, M. J. Pellin, R. S. Lewis, A. M. Davis, R. N. Clayton, S. Amari. Strontium isotopic composition in individual circumstellar silicon carbide grains: A record of $s$-process nucleosynthesis. Phys. Rev. Lett. 81: 3583-3586, 1998.

71) M. J. Pellin, W. F. Calaway, A. M. Davis, R. S. Lewis, S. Amari, R. $\mathrm{N}$. Clayton. Toward complete isotopic analysis of individual presolar silicon carbide grains: $\mathrm{C}, \mathrm{N}, \mathrm{Si}, \mathrm{Sr}, \mathrm{Zr}, \mathrm{Mo}$, and $\mathrm{Ba}$ in single grains of type X. Lunar Planet. Sci. 31: Abstract \#1917, 2000.

72) M. R. Savina, A. M. Davis, C. E. Tripa, M. J. Pellin, R. N. Clayton, R. S. Lewis, S. Amari, R. Gallino, M. Lugaro. Barium isotopes in individual presolar silicon carbide grains from the Murchison meteorite. Geochim. Cosmochim. Acta 67: 3201-3214, 2003.

73) M. R. Savina, A. M. Davis, C. E. Tripa, M. J. Pellin, R. Gallino, R. S. Lewis, S. Amari. Extinct technetium in presolar silicon carbide grains. Science 303: 649-652, 2004.

74) Z. Ma, R. N. Thompson, K. R. Lykke, M. J. Pellin, A. M. Davis. A new instrument for microbeam analysis incorporating submicron imaging and resonance ionization mass spectrometry. Rev. Sci. Instrum. 66: 3168-3176, 1995.

75) B. S. Meyer, D. D. Clayton, L.-S. The. Molybdenum and zirconium isotopes from a supernova neutron burst. Astrophys. J. 540: L49-L52, 2000.

76) P. W. Merrill. Spectroscopic observations of stars of class S. Astrophys. J. 116: 21-26, 1952.

77) T. Stephan, A. M. Davis, M. Pellin, D. Rost, M. R. Savina, R. Trappitsch, N. Liu. CHILI-The final stages of building a challengins instrument. Lunar Planet. Sci. 45: Abstract \#2242, 2014.

78) M. Ishihara, S. Ebata, K. Kumondai, R. Mibuka, K. Uchino, H. Yurimoto. Ultra-high performance multi-turn TOF-SIMS system with a femto-second laser for post-ionization: Investigation of the performance in linear mode. Surf. Int. Anal. 42: 1598-1602, 2010.

79) S. Ebata, M. Ishihara, K. Kumondai, R. Mibuka, K. Uchino, H. Yurimoto. Development of an ultra-high performance multi-turn TOF-SIMS/SNMS system "MULTUM-SIMS/SNMS". J. Am. Soc. Mass Spectrom. 24: 222-229, 2013.

80) M. Toyoda. Development of multi-turn time-of-flight mass spectrometers and their applications. Eur. J. Mass Spectrom. (Chichester, Eng.) 16: 397-406, 2010.

81) K. Terada, M. Nakabayashi, M. Kamioka, M. Toyoda, M. Ishihara, R. Nakamura, J. Aoki, Y. Hino. Development on submicronscale U-Pb dating by laser post-ionized SNMS. in Japan Geoscience Union Meeting 2014. pp. MTT42-P02. 